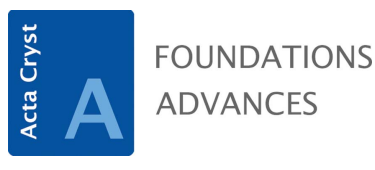

ISSN 2053-2733

\title{
Celebrating 100 years of the Debye scattering equation
}

\author{
Paolo Scardi, ${ }^{a *}$ Simon J. L. Billinge, ${ }^{\mathrm{b}, \mathrm{c} *}$ Reinhard Neder $^{\mathrm{d}}$ and Antonio Cervellino ${ }^{\mathrm{e}}$ \\ ${ }^{a}$ University of Trento, Italy, ${ }^{\mathbf{b}}$ Department of Applied Physics and Applied Mathematics, Columbia University, New York, \\ NY 10027, USA, 'Condensed Matter Physics and Materials Science Department, Brookhaven National Laboratory, \\ Upton, New York, NY 11973, USA, ${ }^{\mathbf{d} F r i e d r i c h-A l e x a n d e r ~ U n i v e r s i t a ̈ t ~ E r l a n g e n-N u ̈ r n b e r g, ~ S t a u d t s t r a s s e ~ 3, ~ D-91058 ~}$ \\ Erlangen, Germany, and ${ }^{\mathbf{e} L a b o r a t o r y}$ for Synchrotron Radiation C, Swiss Light Source, Paul Scherrer Institut, $\mathrm{CH}-5232$ \\ Villigen, Switzerland. *Correspondence e-mail: paolo.scardi@unitn.it, sb2896@columbia.edu
}

Keywords: Editorial; P. Debye; Debye scattering equation; nanomaterials.

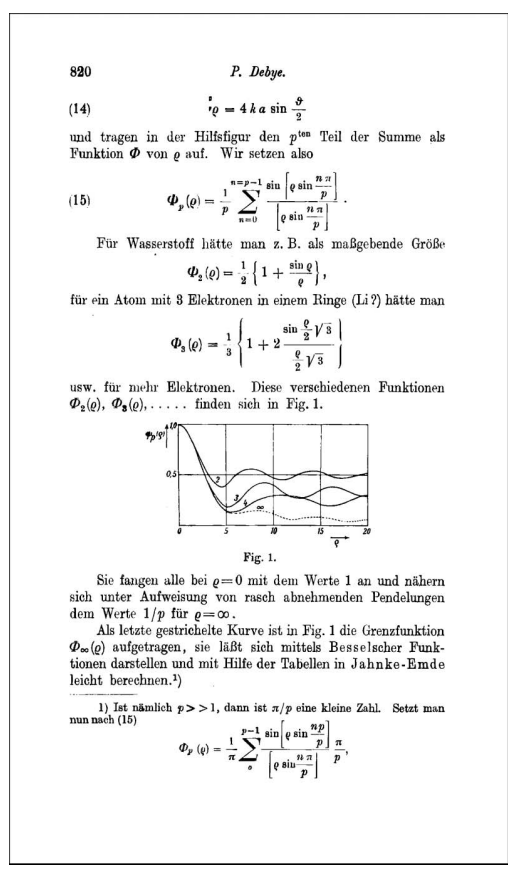

This special issue was commissioned to celebrate a historical event, the first one hundred years of the Debye scattering equation (DSE) (Debye, 1915), also celebrated by a conference held at Cavalese, Italy, 14-18 June 2015 (http://www.dse2015.org/). The Debye scattering equation is a way of calculating the scattered intensity from an isotropic sample, such as a powder, a liquid or an amorphous material, which does not presume periodicity of the underlying structure, the basis of the more famous Bragg and Laue equations. The DSE was written down just two years after these crystallographic equations, but until recently it did not find widespread use. The reasons are twofold. First, for most of the 20th century research was principally focused on crystalline materials. Second, for systems of reasonable size the DSE is very computationally costly to compute. In fact, its first widespread application was in the field of small-angle scattering, where various possible approximations made it an effective and essential analysis tool [Glatter (1977); Glatter \& Kratky (1982); Feigin \& Svergun (1987)]. The situation has now radically changed. There is a huge research effort focused on nanomaterials, and nanostructure in crystalline materials, and computing power has increased such that the DSE can now be computed for systems of a size to be of practical interest. There is therefore enormous growth in applications of the DSE.

Small, non-periodic objects scatter with broad peaks and smoothly varying intensities called diffuse scattering. Modern X-ray synchrotron, neutron and electron diffraction instruments are able to measure the diffuse scattering with good accuracy, using the socalled 'total scattering' approach in which both Bragg (where there are present) and diffuse intensities are measured over wide ranges of reciprocal space. The data may be Fourier transformed to real space, as in the popular atomic pair distribution function (PDF) method, or studied directly in reciprocal space. Either way, for finite objects the DSE is the correct approach to compute the total scattering intensity (and by Fourier transform the PDF) from atomistic models of the cluster under study. The DSE is finding application in many studies in recent years in quite diverse fields of applied sciences ranging from nanotechnology to biology, solid-state physics and chemistry. This special issue presents a small but quite representative snapshot of the various ways in which the DSE is being applied.

Peter Debye's original intuition was that, despite the random arrangement of molecules in gases and liquids, interference between scattering centres can be observed and could reveal details of the atomic arrangement in the molecules. Full exploitation of that had to wait for gas-phase electron diffraction. However, besides contributing to the early understanding of molecular structure, the DSE played a role in the development of fundamental scattering methods, such as the SAXS concept of radius of gyration (Guinier, 1937, 1939), and from the 1930s the pioneering studies on liquids and glasses [e.g., Zachariasen (1932); Warren (1933, 1934, 1940); Zernicke \& Prins (1927)]. Just after the introduction of the first transmission electron microscopes, Germer \& White (1941) showed how to use the DSE to study metal-cluster aggregates; profiting from the regular arrangement of atomic distances in a crystal lattice they recast the DSE in a more computationally efficient form: 


$$
\begin{aligned}
I_{\text {eu }}(q) & =N f^{2}+f^{2} \sum_{i}^{N} \sum_{j \neq i}^{N} \frac{\sin q r_{i j}}{q r_{i j}} \\
& =N f^{2}+f^{2} \sum_{n=1}^{M} \frac{B_{n} \sin q r_{n}}{q r_{n}},
\end{aligned}
$$

where 'eu' indicates electron units. Equation $(1 a)$ is the DSE in the usual form for a monoatomic system (with atomic scattering factor $f$ ), and provides a double sum, over all $N$ atoms with atomic distances $r_{i j}$, with wavevector transfer modulus $q(=4 \pi \sin \theta / \lambda)$; whereas equation ( $1 b)$, proposed by Germer \& White, and still used to date in many applications of the DSE, requires a single sum of the sinc function $\left(\sin q r_{n} / q r_{n}\right)$ weighted on the number $\left(B_{n}\right)$ of atomic pairs separated by the same distance $\left(r_{n}\right)$.

The relationship between the DSE and the autocorrelation function of the density of atoms in real space $\rho(r)$, the founding equation of the PDF approach, was first derived by Zernicke \& Prins (1927):

$$
I_{\text {eu }}(q)=N f^{2}\left\{1+\int 4 \pi r^{2}\left[\rho(r)-\rho_{\mathrm{a}}\right] \frac{\sin q r}{q r} \mathrm{~d} r\right\},
$$

with $\rho_{\mathrm{a}}$ as the average density in the sample. The quantity $4 \pi r^{2} \rho(r)$ is the radial distribution function, representing the deviation from a sample average of the number of scattering centres in a shell of thickness $\delta$ at distance $r_{i}$ from another atom as (Warren, 1990)

$$
Y_{i}=\int_{r_{i}-\delta / 2}^{r_{i}+\delta / 2} 4 \pi r^{2}\left[\rho(r)-\rho_{\mathrm{a}}\right] \mathrm{d} r .
$$

This quantity can be used, if $\rho(r)-\rho_{\mathrm{a}} \neq 0$ over a reasonably short range, to rewrite equation (2) as

$$
I_{\text {eu }}(q) / N=f^{2}+f^{2} \sum_{i} Y_{i} \frac{\sin q r_{i}}{q r_{i}},
$$

establishing the sine Fourier relationship between the DSE and the radial distribution function and the PDF.

So, it is a great moment in time to have a special issue dedicated to the Debye scattering equation: the DSE just turned 100 years old, but it is now being used more than ever, two causes for celebration. We thank the contributing authors for their contributions, and we very much hope that you enjoy the special issue. Perhaps one day soon you will use the DSE in your own work, if you haven't already!

\section{Acknowledgements}

SJLB acknowledges support from US Department of Energy, Basic Energy Sciences, through grant DE-SC0012704.

\section{References}

Debye, P. J. W. (1915). Ann. Phys. 351, 809-823.

Feigin, L. A. \& Svergun, D. I. (1987). Structure Analysis by Small Angle $X$-ray and Neutron Scattering. New York, London: Plenum Press.

Germer, L. H. \& White, A. H. (1941). Phys. Rev. 60, 447-454.

Glatter, O. (1977). J. Appl. Cryst. 10, 415-421.

Glatter, O. \& Kratky, O. (1982). Small-Angle X-ray Scattering. New York: Academic Press.

Guinier, A. (1937). C. R. Acad. Sci. 204, 1115.

Guinier, A. (1939). Ann. Phys. 12, 161.

Warren, B. E. (1933). Z. Kristallogr. 86, 349.

Warren, B. E. (1934). Phys. Rev. 45, 657-661.

Warren, B. E. (1940). Chem. Rev. 26, 237-255.

Warren, B. E. (1990). X-ray Diffraction, pp. 151-201. Reading: Dover

Zachariasen, W. H. (1932). J. Am. Chem. Soc. 54, 3841-3851.

Zernike, F. \& Prins, J. A. (1927). Z. Phys. 41, 184-194. 